\title{
Cocrystals of Leflunomide: Design, Structural and Physicochemical Evaluation
}

\author{
Joseph Cadden, ${ }^{\dagger,}$ Wim T. Klooster, ${ }^{\ddagger}$ Simon J. Coles, ${ }^{\ddagger}$ and Srinivasulu Aitipamula, ${ }^{, \dagger}$ \\ ${ }^{\dagger}$ Crystallisation and Formulation Science, Institute of Chemical and Engineering Sciences, \\ A*STAR (Agency for Science, Technology and Research), 1 Pesek Road, Jurong Island, \\ 627833, Singapore. \\ ${ }^{\ddagger}$ Chemistry, Faculty of Natural and Environmental Sciences, University of Southampton, \\ University Road, Southampton SO17 1BJ, U.K.
}

\begin{abstract}
The use of cocrystallisation as a tool to improve the pharmaceutical profile of the low-solubility drug leflunomide, used in the treatment of arthritis, is herein evaluated. Judicious selection of coformers based upon knowledge-based strategy and crystal engineering principles has resulted in new cocrystals with pyrogallol, 3-hydroxybenzoic acid, 2-picolinic acid, and 2-aminopyrimidine. Characterisation and structure determination of these systems was performed using X-ray diffraction. Crystal structure analysis revealed that the hydrogen bonding in the crystal structures corroborate well with the knowledge-based prediction tool. Physicochemical properties such as thermal behaviour, stability, solubility, and dissolution rate of the pharmaceutically acceptable cocrystals were evaluated to assess their impact on the pharmaceutical profile of leflunomide.
\end{abstract}


When compared with their parent compound leflunomide and the physical mixtures, cocrystals were found to exhibit improved physicochemical properties, showing their potential for development of new solid dosage forms.

\section{INTRODUCTION}

With the advent of novel and sophisticated drug discovery technologies, a large number of active pharmaceutical ingredients (APIs) are being discovered and tested for their therapeutic applications. ${ }^{1}$ However, fresh challenges are being faced in the development of single-component crystalline APIs into solid dosage forms due to the fact that majority of these actives belong to class II, III, and IV of the Biopharmaceutical Classification System (BCS), where bioavailability is limited by low solubility or permeability., $\underline{2}$ Up to $40 \%$ of newly discovered pharmaceutical compounds are hydrophobic, and thus have their pharmacological activity limited by poor solubility, $\stackrel{4}{ }$ a problem that is likely to worsen with potentially between 70 and $90 \%$ of current drug candidates predicted to possess low solubility. 4

There are a variety of commonly employed techniques to counter this problem, such as particle size reduction,,$\frac{5}{}$ amorphisation,,$\underline{6}$ complexation using cyclodextrins, $\stackrel{\underline{7}}{-}$ solid dispersion, $\stackrel{\underline{8}}{\text { etc. }}$ The use of multi-component systems is a viable and widely used tool for improving solubility profile of a crystalline API. Conventional design of crystalline forms of APIs within a multicomponent system involves, but is not limited to, the use of salts, solvates and hydrates. ${ }^{9}$ While cocrystals are well recognised as a class of systems utilised for the manipulation and combination of the properties of different components into a single crystal lattice, their use represents a newly established and adopted approach to synthesising crystalline materials in the pharmaceutical industry. ${ }^{10}$ Marketable pharmaceutical cocrystals are generally comprised of an API and one or 
more inactive cocrystal formers (coformers) that are solids under ambient conditions. ${ }^{10}, \underline{11} \mathrm{~A}$ coformer can also be another API, resulting in multi-API cocrystal for synergistic effects on drug properties. ${ }^{12}$ The current interest in pharmaceutical cocrystals is due to the ability of coformers to modify physicochemical properties of the API such as solubility, stability and bioavailability without making or breaking covalent bonds, or affecting its pharmacological activity. $\underline{\underline{11}} \underline{13}$ Although restricted to those that are Generally Regarded as Safe (GRAS), the availability of a wide range of GRAS coformers can lead to many potential cocrystals for any particular API. $\underline{14}$

Traditionally, the search for, and discovery of viable new pharmaceutical cocrystals is performed via high-throughput (HT), or random screening by solid-state grinding and crystallisation, whereby only a handful of "hits" may be generated from an initial selection of many candidates. $\cdot \underline{15}$ Current literature show efforts to improve and streamline the cocrystal screening process. $\stackrel{16}{ }$ By using a priori design and selection of coformers; employing the principles of crystal engineering alongside a library of crystal structures and intermolecular interactions, such as the Crystal Structure Database (CSD),,$\underline{17}$ a variety of methods for logical coformer selection can be employed. These include hydrogen bond propensity calculations (HBPCs), $\frac{18}{}$ and similar twostage rational selection of coformers based on structural diversity, $\underline{\underline{19}}$ and the use of known structurally similar compounds to enable the identification of appropriate coformers for cocrystal screening. $\underline{20}, \underline{21}$

Leflunomide (5-methyl-N-[4-(trifluoromethyl) phenyl]-isoxazole-4-carboxamide, LEF, Figure 1) is an immunosuppressive disease-modifying antirheumatic drug that is used to slow down progression of the rheumatoid arthritis through inhibition of pyrimidine synthesis. ${ }^{22}$ It is formulated as $\operatorname{Arava}^{\circledR}$, and is converted into its active metabolite, Teriflunomide in vivo. Possessing low water solubility (less than $40 \mathrm{mg} \mathrm{L}^{-1}$ ), LEF belongs to the BCS class II of drugs. $\underline{23}$ 
These properties, along with the notable clinical efficacy of LEF, make cocrystallisation an attractive prospect for enhancing its drug formulation, absorption and delivery properties through increased solubility. Vega et al. ${ }^{24}$ have reported two polymorphic forms of LEF. Both polymorphs (Forms I and II) crystallise in the monoclinic space group $P 2_{1} / c$ with $Z^{\prime}$ values of 2 and 1 , respectively, and Form I being the thermodynamically stable form at room temperature..$^{24}$ The presence of amide and isoxazole functional groups in LEF enables formation of a number of possible supramolecular synthons ${ }^{25}$ with complimentary functional groups for synthesis of multicomponent systems.

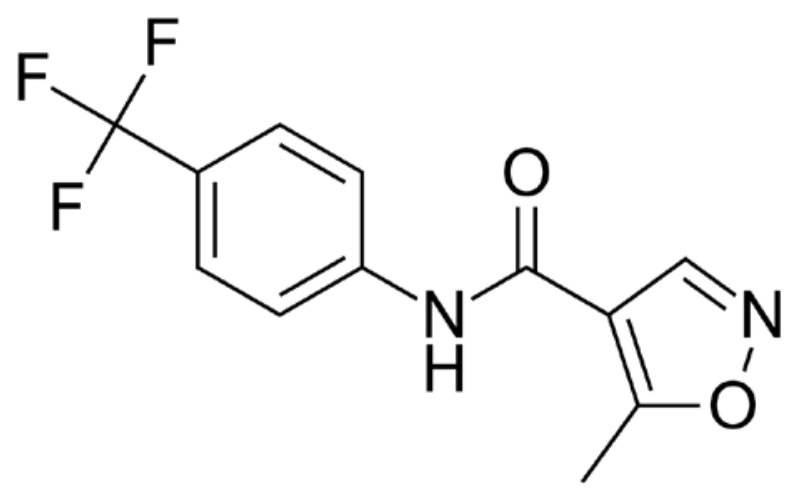

Figure 1. Chemical diagram of LEF with atomic numbering scheme.

This study aimed to use a knowledge-based strategy for selection and synthesis of cocrystals of LEF with a view to evaluate their pharmaceutical profile. Experiments have yielded four pharmaceutically acceptable cocrystals (along with a cocrystal with a non-GRAS coformer). ${ }^{26,}{ }^{27}$ We herein report their characterisation by powder and single-crystal X-ray diffraction and thermal analysis, along with the evaluation of their physicochemical properties. 


\section{EXPERIMENTAL SECTION}

LEF was purchased from Biotain Pharma Co., Ltd., China, while all coformers were purchased from Sigma-Aldrich, Singapore, and used as received without any further purification. Analytical grade solvents were used for the crystallisation experiments.

Solid-State Screening. Cocrystal screening by grinding was performed via solvent drop grinding using a Retsch Mixer Mill model MM301. In each experiment, $270.2 \mathrm{mg}$ (0.1 mmol) of LEF, a stoichiometric amount of the coformer and 2 drops of methanol were added to stainless steel grinding jars $(10 \mathrm{~mL})$ with one $7 \mathrm{~mm}$ stainless steel grinding ball and ground at a rate of $20 \mathrm{~Hz}$ for $20 \mathrm{~min}$. The resultant products were characterised by powder X-ray diffraction (PXRD) and compared with the reference starting materials for identification of a promising new solid form.

Solvent Based Cocrystallisation. Solution crystallisation by solvent evaporation was used to prepare single crystals of the new solid forms identified as promising from the grinding experiments. These were used in single crystal X-ray diffraction experiments.

LEF-PG cocrystal. LEF (270.2 mg, $0.1 \mathrm{mmol}$ ) and PG (126.1 mg, $0.1 \mathrm{mmol}$ ) were dissolved in chloroform $(10 \mathrm{~mL})$ at $50{ }^{\circ} \mathrm{C}$ and the solution left for evaporation at ambient conditions. After 12 days large needle-like crystals were formed.

LEF-3HBA cocrystal. LEF (270.2 mg, $0.1 \mathrm{mmol})$ and 3HBA (138.1 mg, $0.1 \mathrm{mmol})$ were dissolved in acetic acid $(10 \mathrm{~mL})$ at $90{ }^{\circ} \mathrm{C}$ and the solution left for evaporation at ambient conditions. After 2 weeks small needle-like crystals were formed. 
LEF-2PIC cocrystal. LEF (270.2 mg, $0.1 \mathrm{mmol}$ ) and PIC (123.1 mg, $0.1 \mathrm{mmol}$ ) were dissolved in methanol $(5 \mathrm{~mL})$ at $50{ }^{\circ} \mathrm{C}$ and the solution left for evaporation at ambient conditions. Large block-shaped crystals were obtained after 2-3 days.

LEF-2PIC (1:2) cocrystal. LEF (270.2 mg, $0.1 \mathrm{mmol}$ ) and PIC (246.2 mg, $0.2 \mathrm{mmol}$ ) were dissolved in methanol $(10 \mathrm{~mL})$ at $50^{\circ} \mathrm{C}$ and the solution left for evaporation at ambient conditions. After 5-6 days small whitish needle-like crystals were formed.

LEF-2APYM cocrystal. LEF (270.2 mg, $0.1 \mathrm{mmol}$ ) and 2APYM (95.1 mg, $0.1 \mathrm{mmol}$ ) were dissolved in methanol (5 mL) at $50{ }^{\circ} \mathrm{C}$ and the solution left for evaporation at ambient conditions. After 2-3 days large block-shaped crystals were formed.

Powder X-ray Diffraction (PXRD). PXRD data for samples generated through solid-state grinding, stability, and slurry experiments were collected using a Bruker D8 Advance powder Xray diffractometer with $\mathrm{Cu}-\mathrm{K} \alpha$ radiation $(\lambda=1.54060 \AA)$, with $35 \mathrm{kV}$ and $40 \mathrm{~mA}$ voltage and current applied. The sample was scanned within the range $2 \theta=5^{\circ}$ to $50^{\circ}$ with continuous scanning mode, and a scan rate of $5^{\circ} \mathrm{min}^{-1}$.

Single Crystal X-ray Diffraction. Data for all cocrystals were collected on an Agilent Technologies Dual Source Supernova, four-circle diffractometer fitted with CCD detector, with graphite monochromated Mo-K $\alpha$ radiation $(\lambda=0.71073 \AA)$ for the LEF-PG, LEF-2PIC and LEF2APYM cocrystals, and Cu-K $\alpha$ radiation $(\lambda=1.54184 \AA)$ for the LEF-3HBA and LEF-2PIC 1:2 cocrystals. CrysAlisPro software ${ }^{28}$ was used for data collection, reduction, and absorption correction using face indexing and Gaussian corrections. Structure solution and refinement were carried out using Intrinsic Phasing in SHELXT-2015 29 and refined by full-matrix least squares on 
F2 using SHELXL-2015, ${ }^{30}$ both implemented in the Olex2 software. ${ }^{31}$ Non-hydrogen atoms in all structures were refined with anisotropic displacement parameters. Hydrogen atoms for heteroatom ( $\mathrm{N}$ and $\mathrm{O}$ ) were located from the difference Fourier map and refined freely, maintaining isotropic displacement parameters $\left(\mathrm{U}_{\mathrm{iso}}\right)$ whilst the remaining hydrogen atoms were fixed in idealised positions with their displacement parameters riding on the values of their parent atoms. Hydrogen bond lengths were neutron normalised (for $\mathrm{O}-\mathrm{H}, \mathrm{N}-\mathrm{H}$ and $\mathrm{C}-\mathrm{H}$ at $0.983 \AA, 1.009 \AA$ and $1.008 \AA$, respectively) using PLATON ${ }^{32}$ for the calculation of bond lengths and bond angles. X-Seed ${ }^{33}$ was used for the preparation of crystal structure figures. Crystal structure parameters and hydrogen bond parameters for all structures presented can be found in the Supporting Information. CCDC 1901849-1901853 contains the supplementary crystallographic data for this paper. These data can be obtained free of charge from The Cambridge Crystallographic Data Centre via http://www.ccdc.cam.ac.uk/conts/retrieving.html and deposited as Supporting Information.

The crystal structure of the LEF-PG cocrystal presented herein is modelled in the $I-4$ space group. Examination of the raw data indicates a degree of commensurate modulation. After testing a variety of spacegroups, from triclinic to tetragonal crystal systems, it was decided that the $I-4$ space group symmetry gives a model which is a close approximation to the true commensurate structure (for more details see the Supporting Information, Figures S14-16).

Differential Scanning Calorimetry (DSC). A Mettler Toledo DSC 822e module was used for the DSC analysis. In each experiment, the sample size was $2-5 \mathrm{mg}$, the temperature range was typically $25-250^{\circ} \mathrm{C}$, and the heating rate was $10^{\circ} \mathrm{C} \mathrm{min}^{-1}$. The samples were purged with a stream of flowing nitrogen $\left(20 \mathrm{~mL} \mathrm{~min}^{-1}\right)$. The instrument was calibrated using Indium as the reference material. 
Stability Studies. The stability of all the pharmaceutically acceptable cocrystals was evaluated under accelerated storage condition $\left(40^{\circ} \mathrm{C}\right.$ and $75 \%$ relative humidity (RH)) over a period of 13 weeks in an MMM Climacell 111 humidity incubator. Samples of approximately $100 \mathrm{mg}$ were stored under the test condition and tested periodically using PXRD to identify the samples' identity.

For the slurry experiments, excess powder samples of the cocrystals were left stirring in ultrapure water at $37^{\circ} \mathrm{C}$ for $24 \mathrm{~h}$ before filtering and drying in ambient conditions, and the resulting powders were analysed by PXRD.

Solubility Studies. Solubility was measured by stirring excess amounts of powder samples in 5 $\mathrm{mL}$ of ultrapure water at $37^{\circ} \mathrm{C}$ for $24 \mathrm{~h}$. Upon equilibration, the samples were filterred through a $45 \mu \mathrm{m}$ syringe filter and concentration of the aliquots was quantified by HPLC using predetermined calibration data.

Dissolution Studies. The dissolution rate of all samples (LEF, cocrystals and physical mixtures) was tested using an Agilent 708-DS dissolution sampling apparatus. For all the powder dissolution experiments, samples containing equivalent to $25 \mathrm{mg}$ of LEF in a $500 \mathrm{mg}$ corn starch tablet were pressed for $30 \mathrm{~s}$ at $1 \mathrm{kN}$ using an FT-IR press. Corn starch was used as a disintegration agent which also faciliated thorough distribution of powders in the dissolution vessel. Ultrapure water was used as dissolution medium $(900 \mathrm{~mL})$ with a rotation speed of $100 \mathrm{rpm}$ at $37^{\circ} \mathrm{C}$. All the tablets were found to disintegrate within a minute. For Intrinsic Dissolution Rate (IDR) experiments, approximately $100 \mathrm{mg}$ of the sample was compressed to a $0.5 \mathrm{~cm}^{2}$ disk in a rotating disk intrinsic dissolution die using a hydraulic press at a pressure of 2 tons for 2 min such that only one side of the disk was exposed to the dissolution medium and the surface of the disk was constant throughout the experiment. Sampling was conducted by withdrawing $2 \mathrm{~mL}$ aliquots of sample from the vessel 
and filtered through a $45 \mu \mathrm{m}$ syringe tip filter for analysis by HPLC. This was conducted at 5, 10, 15, 30, 45, 60, 90, 120, 180, 240, 300 and 360 mins. In order to maintain the dissolution volume, samples were immediately replaced with an equivalent fresh dissolution medium. All the dissolution experiments were run in triplicate. The apparent solubility values were calculated using the dissolution data from the linear portion of the dissolution profile.

High Performance Liquid Chromatography (HPLC). HPLC was used to quantify LEF concentration in the samples obtained from solubility and dissolution experiments. HPLC instrument was equipped with a ZORBAX ECLIPSE XDB-C18 column (4.6 mm x $250 \mathrm{~mm}$ x 5 $\mu \mathrm{m})$ and run at $37^{\circ} \mathrm{C}$. The mobile phase consisted of acetonitrile and water (50:50 v/v) with a flow rate of $1 \mathrm{~mL} \mathrm{~min}{ }^{-1}$ and $15 \min$ run time. A sample injection volume of $50 \mu \mathrm{L}$ was used. The detection wavelength was set to $262 \mathrm{~nm}$. The retention time of LEF was 7.4 mins, and the retention times of the coformers PG, 3HBA and 2PIC were 2.6, 2.8 and 2.2 mins, respectively.

\section{RESULTS AND DISCUSSION}

Interaction Searching. Propensity of the functional groups present in LEF to interact with other complementary functional groups can be understood through statistical analysis of the crystal structures deposited in the CSD. Selecting "ligands", representative of the functional groups in LEF, and possible contact groups from a pre-set list available in Isostar v 2.2.5 $5^{34}$ enabled the determination of the most preferential contacts for each functional group. LEF contains a number of functional groups with potential for intermolecular interactions, mainly an amide group (bonded either side to aromatic rings), an isoxazole ring (methyl substituted), and a trifluoromethyl group. These were initially modelled using selected ligands available in the Isostar library: aromatic- 
aromatic amide, isoxazole, aromatic $\mathrm{O}$, aromatic $\mathrm{N}$ in 5-membered rings, trifluoromethyl, and were labelled the central groups (see Supporting Information). Isostar searching based on these central groups, with respect to a variety of common functional groups (contact groups), gave statistical information on the number of crystal structures containing both the central and contact groups, and the number of these structures in which the central and contact groups form a contact with distance $(R)$ less than the sum of the van der Waals' radii $(V)$. Detailed results of these searches can be found in the Supporting Information.

Statistical analysis of intermolecular contacts in the Isostar allowed for a primary evaluation of the preferred interactions between the functional groups of LEF and potential coformers. All five aforementioned contact groups showed a general high affinity to form contacts with groups containing an aromatic component, such as $\mathrm{C}(\mathrm{ar})-\mathrm{NH}_{2}$, aromatic $\mathrm{C}-\mathrm{H}$, and phenol $\mathrm{O}-$ $\mathrm{H}$ groups. In addition to these, polar $\mathrm{X}-\mathrm{H}$ groups $(\mathrm{X}=\mathrm{N}, \mathrm{O}$ or $\mathrm{S}$ ) were amongst the highest occurring contacts for all the central groups, with specifically $\mathrm{OH}$ group showing a high affinity for contact with the aromatic-aromatic amide, isoxazole and aromatic $\mathrm{N}$ central groups, and $\mathrm{N}-\mathrm{H}$ group likewise for the aromatic $\mathrm{N}$ and trifluoromethyl central groups. This leads to three initial preferences in coformer structure and functional groups for the design of LEF cocrystals: O-H group, and either amide or amine $\mathrm{N}-\mathrm{H}$ groups.

With Isostar interaction search providing an initial insight into the nature and preference of interactions in the various functional groups of LEF, specific contact searching was then conducted using the motif search tool integrated in Mercury. ${ }^{35}$ Rather than investigating contacts between predefined ligands and groups, each functional group could be tailored to more accurately represent those involved in LEF and its possible contacts (see Supporting Information). In addition to this, the nature of interactions could be defined, such as separation into donor and acceptor 
atoms. Similar to the Isostar interaction search, the dominant hydrogen bonding motifs from the various LEF functional groups involved amide, various aliphatic and aromatic hydroxyl (including $\mathrm{COOH}$ ) and amine contact groups. The results of these specific contact searches are described in detail in the Supporting Information.

Molecular Complementarity and Selection of Coformers. Based on the most frequently occurring intermolecular interactions, involving central groups representative of those in LEF and a variety of contact groups, a library of favourable functional groups for cocrystal design was formed. These functional groups include primary amine, amide, and hydroxyl groups, with an additional preference for aromaticity when $\mathrm{C}-\mathrm{H}$ groups are considered. Interestingly, these functional groups represent the possibility for formation of a number of well-known supramolecular synthons, such as $\mathrm{O}-\mathrm{H} \cdots \mathrm{N}(\mathrm{ar})$ and $\mathrm{O}-\mathrm{H} \cdots \mathrm{CONH}_{2} \cdot{ }^{36}$ In addition to this, the potential for $\mathrm{COOH} / \mathrm{hydroxyl}-\mathrm{N}(\mathrm{ar})$ heterosynthon formation involving the isoxazole moiety of LEF provides an argument for carboxylic acid functional groups to be considered..$^{37}$

Using the knowledge of these preferred functional groups, a number of coformers were chosen from a list of those commonly used in cocrystal screening experiments, ensuring a wide range of structures to encompass the varying types of motifs shown to be favourable. These were checked against a library of coformers from the CSD which are provided within the molecular complementarity search tool implemented in Mercury. Any coformers that were not already present within this library, but used in the cocrystal screening, were manually added. The molecular complementarity search uses analysis of properties such as coformer shape, size and polarity, amongst other factors, to generate a list of potential hits for a particular molecule. Results of these predictions in turn allowed selection of coformers for the solid form screening of LEF. 
Consistent with those from the interaction searches, these results predicted a high complementarity of LEF with aromatic $\mathrm{O}-\mathrm{H}, \mathrm{N}-\mathrm{H}$ and heteroatom containing coformers, as well as a number of dicarboxylic acids. Details of the coformers searched and those that predicted cocrystal formation can be found in the Supporting Information.

Solid Form Screening. Judicious selection of coformers based on the in-depth CSD analysis of LEF interactions and application of prediction tools followed by solid-form screening using solvent drop grinding methods yielded a total of five new cocrystals (see Supporting Information, Figures S5-13). Three of these coformers, 2-picolinic acid (2PIC), 2-aminopyrimidine (2APYM) and 3-hydroxybenzoic acid (3HBA) were identified as hits from the molecular complementarity search, with pyrogallol (PG) falling into the fail list. The chemical structures of these coformers are shown in Figure 2.<smiles>Oc1cccc(O)c1O</smiles>

pyrogallol

PG<smiles>Nc1ncccn1</smiles>

2-aminopyrimidine 2APYM<smiles>O=C(O)c1cccc(O)c1</smiles>

3-hydroxybenzoic acid 3HBA<smiles>O=C(O)c1ccccn1</smiles>

2-picolinic acid 2PIC

Figure 2. Chemical diagram of the coformers that formed cocrystals with LEF. 
Crystal Structure Analysis. LEF-PG Cocrystal. Crystal structure of LEF-PG cocrystal belongs to the tetragonal I-4 space group with the asymmetric unit containing each one molecule of LEF and PG. The crystal structure features hydrogen bonded chains of PG molecules via $\mathrm{O}-\mathrm{H} \cdots \mathrm{O}$

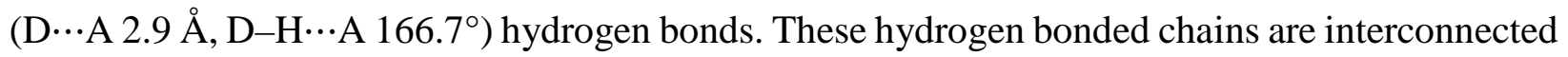
to each other by LEF molecules, stacked along the crystallographic $c$-axis via $\pi \cdots \pi$ interactions, involving $\mathrm{O}_{\mathrm{PG}}-\mathrm{H} \cdots \mathrm{O}_{\mathrm{LEF}}\left(2.7 \AA, 136.6^{\circ}\right)$ and $\mathrm{N}_{\mathrm{LEF}}-\mathrm{H} \cdots \mathrm{O}_{\mathrm{PG}}\left(3.0 \AA, 138.4^{\circ}\right)$ hydrogen bonds. These LEF molecules are stacked in an alternating orientation forming an ABAB type of arrangement. Within this alternating framework of LEF molecules, the 'A' molecules form $\mathrm{O}-\mathrm{H} \cdots \mathrm{O}$ and $\mathrm{N}-$ $\mathrm{H} \cdots \mathrm{O}$ hydrogen bonds with PG molecules above and below the plane, respectively, and vice versa for the 'B' molecules of LEF (Figure 3).

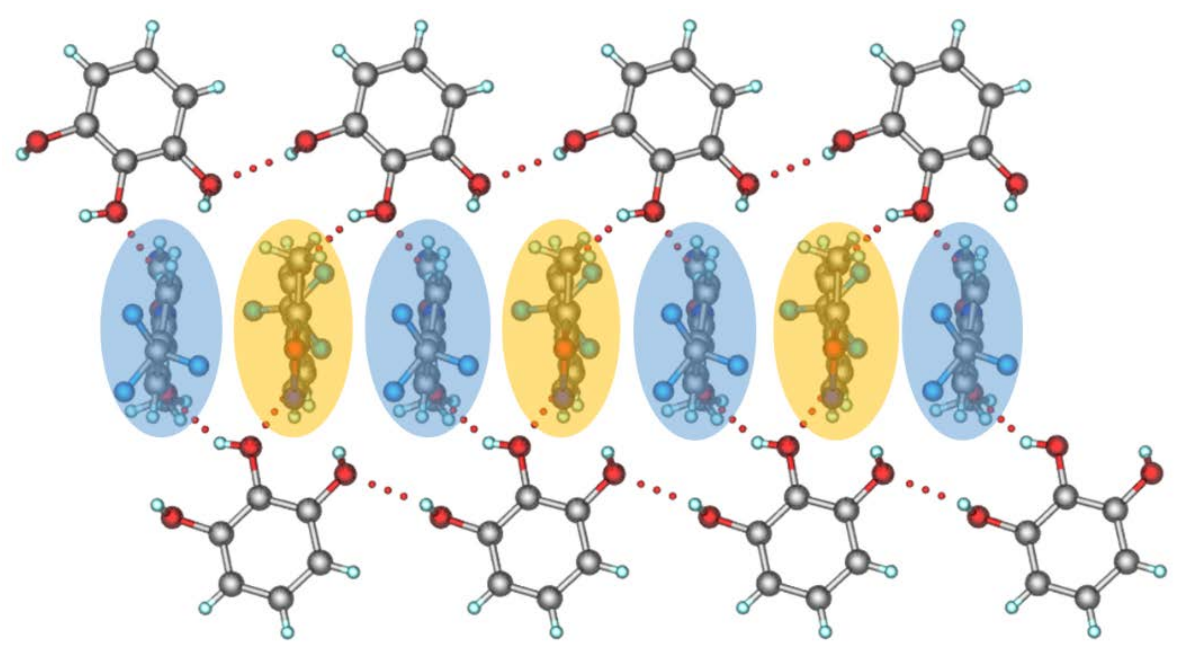

Figure 3. Crystal structure of the LEF-PG cocrystal viewed along the $(a+b) / 2$ axis, showing the primary hydrogen-bonding interactions along with the alternating $\mathrm{ABAB}$ type orientation of LEF molecules. ' $\mathrm{A}$ ' molecules are shown in blue with ' $\mathrm{B}$ ' molecules shown in orange.

LEF-3HBA Cocrystal. The LEF-3HBA cocrystal contains each one molecule of LEF and 3HBA in the asymmetric unit and features a mixture of hydroxyl-acid, acid-isoxazole and amideamide interactions. Self-assembly of LEF molecules via amide-amide $\mathrm{N}-\mathrm{H} \cdots \mathrm{O}\left(2.976 \AA\right.$, $\left.156.8^{\circ}\right)$ 
hydrogen bond along the crystallographic $b$-axis generate hydrogen bonded chains. 3HBA molecules also self-assemble to form hydrogen bonded chains via $\mathrm{O}-\mathrm{H} \cdots \mathrm{O}\left(2.735 \AA, 1^{168.7^{\circ}}\right)$ hydrogen bond involving hydroxyl and carbonyl groups. These chains consist of 3HBA molecules in alternating orientations connected either side by acid-isoxazole $\mathrm{O}-\mathrm{H} \cdots \mathrm{N}\left(2.784 \AA ⿻ 174.2^{\circ}\right)$ hydrogen bonding to LEF chains that extend parallel along the $b$-axis through amide-amide $\mathrm{N}-$

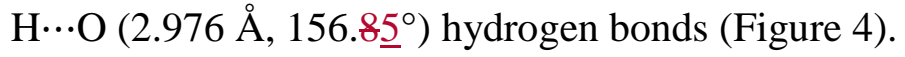
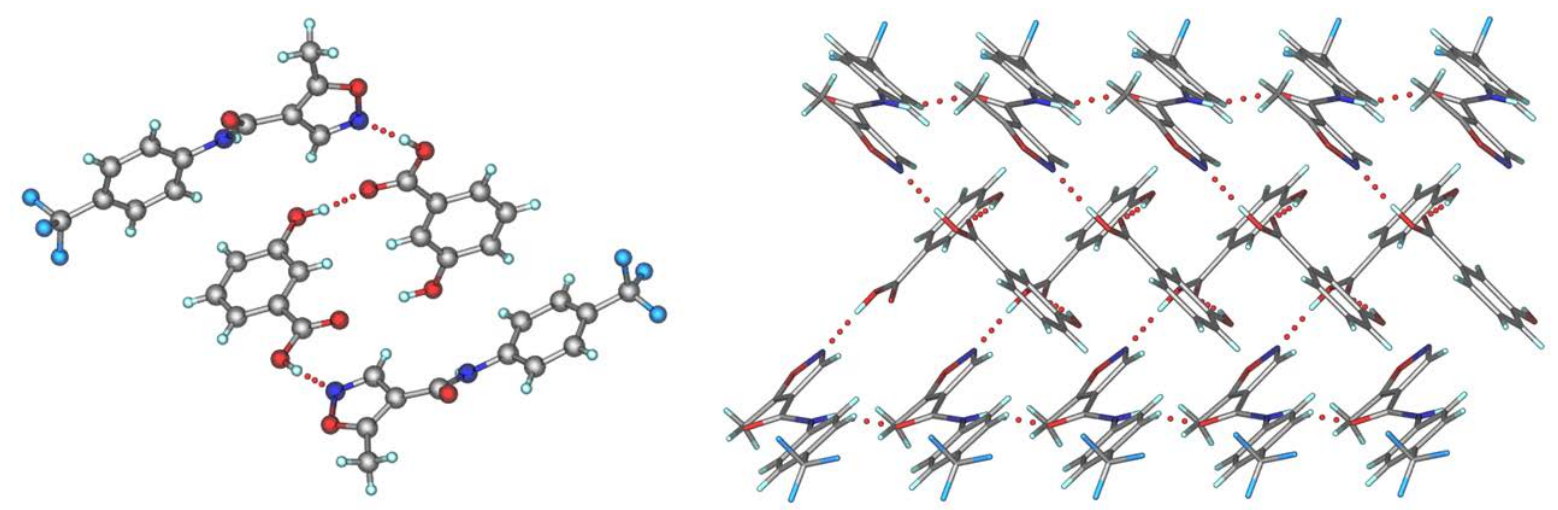

Figure 4. Left, LEF-3HBA viewed down the b-axis showing the hydrogen bonding between components. Right, LEF-3HBA chains viewed down the $(a+c) / 2$ axis showing the alternating orientation of 3HBA molecules.

LEF-2PIC Cocrystal. Two different cocrystals were obtained from cocrystallisation of LEF and 2PIC. The first being a 1:1 cocrystal (hereafter referred to as LEF-2PIC, with 1:1 stoichiometry implied), with an intramolecular proton transfer occurring within 2PIC from O3 to N3. This proton transfer enables formation of coformer carboxylate-pyridinium dimers through charge-assisted $\mathrm{N}^{+}-\mathrm{H} \cdots \mathrm{O}^{-}\left(2.666 \AA, 15 \underline{1} \theta .7 \underline{4}^{\circ}\right)$ hydrogen bonds. These dimers connect inversion related LEF molecules through amide-carboxylate $\mathrm{N}-\mathrm{H} \cdots \mathrm{O}^{-}\left(2.893 \AA\right.$, $\left.159 . \underline{6} 7^{\circ}\right)$ hydrogen bond and $\mathrm{C}-\mathrm{H}_{2 \mathrm{PIC}} \cdots \mathrm{O}_{\mathrm{LEF}}$ interaction and generate ladder network along the crystallographic $c$-aixs where the LEF molceules made up the rungs while 2PIC dimers make the ladder steps (Figure 5). 


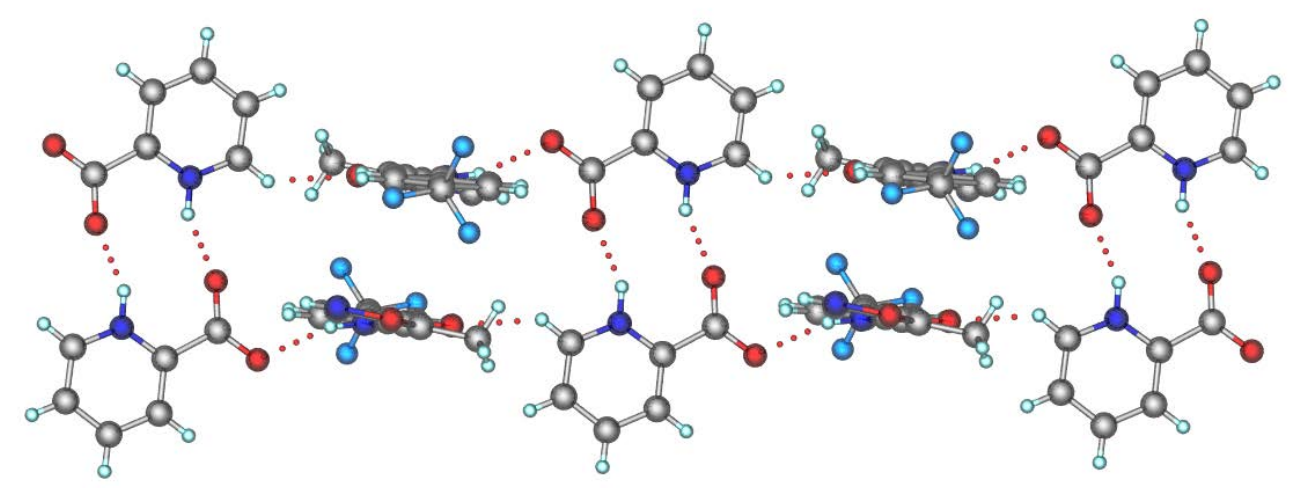

Figure 5. Crystal structure of the LEF-2PIC cocrystal viewed down the $(a+b) / 2$ axis, showing the self-assembly of LEF and 2PIC as hydrogen bonded ladders along the crystalographic $c$-axis.

LEF-2PIC 1:2 Cocrystal. LEF also forms a 1:2 cocrystal with 2PIC (hereafter LEF-2PIC (1:2)), again with the 2PIC molecule exhibiting an intramolecular proton transfer from the carboxylic acid group to the pyridine nitrogen. This affords two charge-assisted $\mathrm{N}^{+}-\mathrm{H}^{\cdots} \mathrm{O}^{-}(2.649$ $\AA$, $159.3 \underline{5}^{\circ} ; 2.7043 \AA \AA, 157 \underline{9} .9 \underline{5}^{\circ}$ ) hydrogen bonds between the symmetry independent 2PIC molecules possessing alternating orientations orthogonal to each other. This results in hydrogen bonded chains of 2PIC along the crystallographic c-axis. These chains are linked to each other by LEF molecules via amide-carboxylate $\mathrm{N}-\mathrm{H} \cdots \mathrm{O}^{-}\left(2.848 \AA \AA \AA, 168.9 \underline{8}^{\circ}\right)$ hydrogen bonds and auxiliary $\mathrm{C}-\mathrm{H}_{2 \mathrm{PIC}} \cdots \mathrm{O}_{\mathrm{LEF}}$ interactions (Figure 6) and generate hydrogen bonded strands along the crystallographic $b$-axis. The inversion related such strands self assemble in the crystal structures via $\pi \cdots \pi$ interactions. 

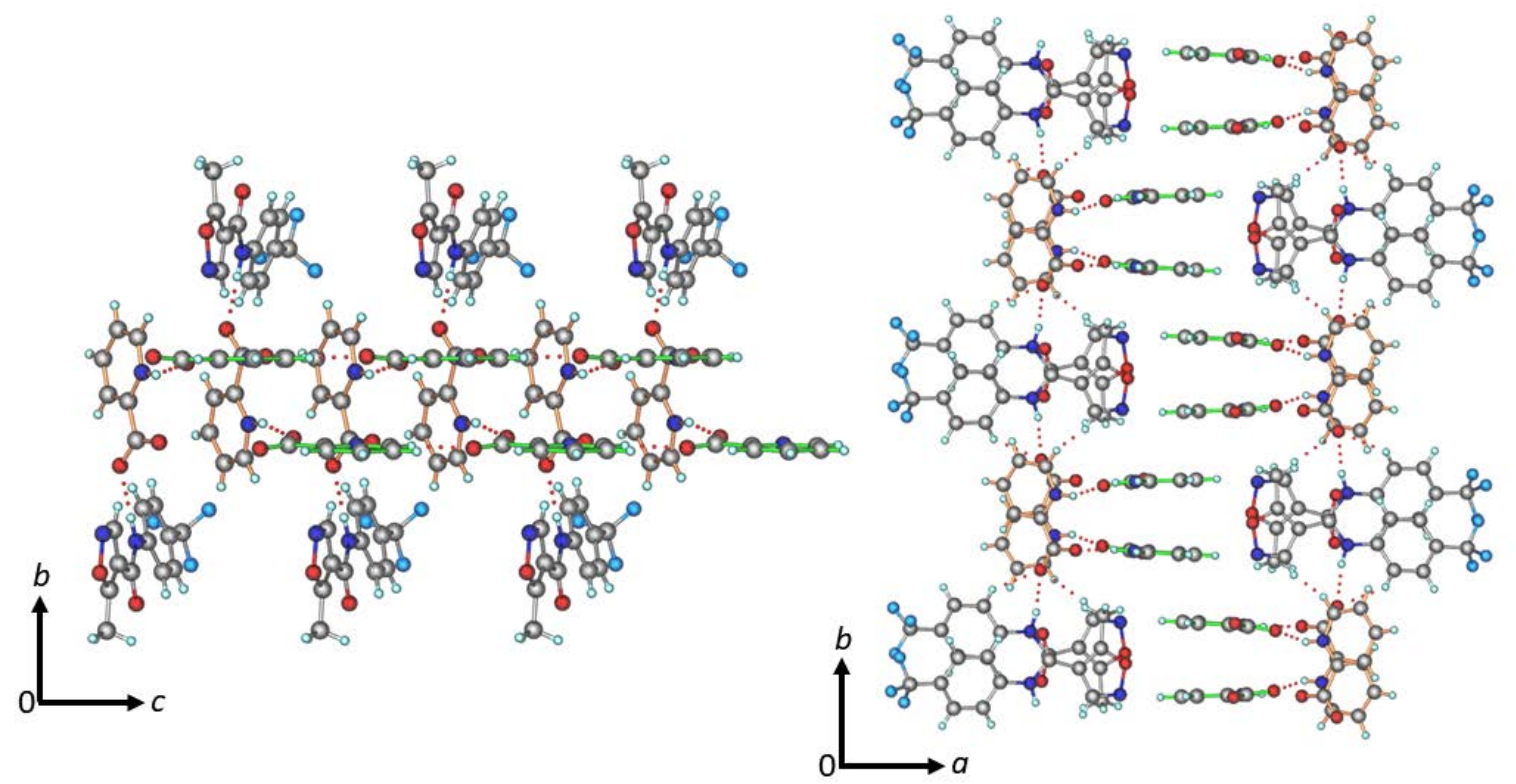

Figure 6. Crystal structure of the LEF-2PIC 1:2 cocrystal showing a single hydrogen bonded strand (left) and the arrangement of inversion related hydrogen bonded strands (right).

LEF-2APYM Cocrystal. LEF and 2APYM form a 1:1 cocrystal featuring a primary

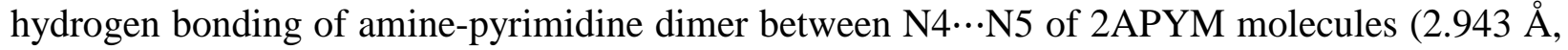
$176.0^{\circ}$ ). Each $2 \mathrm{APYM}$ molecule also interacts separately with two LEF molecules through amide $\mathrm{N}-\mathrm{H} \cdots$ pyrimidine $\left(3.072 \AA, 166.6^{\circ}\right)$ and amine $\mathrm{N}-\mathrm{H} \cdots$ amide $\mathrm{O}=\mathrm{C}\left(2.941 \AA, 127.7^{\circ}\right)$ hydrogen bonds. These result in a crystal packing similar to that of the LEF-2PIC 1:1 cocrystal, whereby 2APYM-2APYM dimers connect LEF molecules that stack above each other in inverted orientations. However, unlike the LEF-2PIC 1:1 cocrystal, four-membered supramolecular unit is not formed in LEF-2APYM cocrystal due to the fact that the amide $\mathrm{C}=\mathrm{O}$ of LEF molecule helps to form an extended hydrogen-bonded network along the crystallographic $b$-axis (Figure 7). 


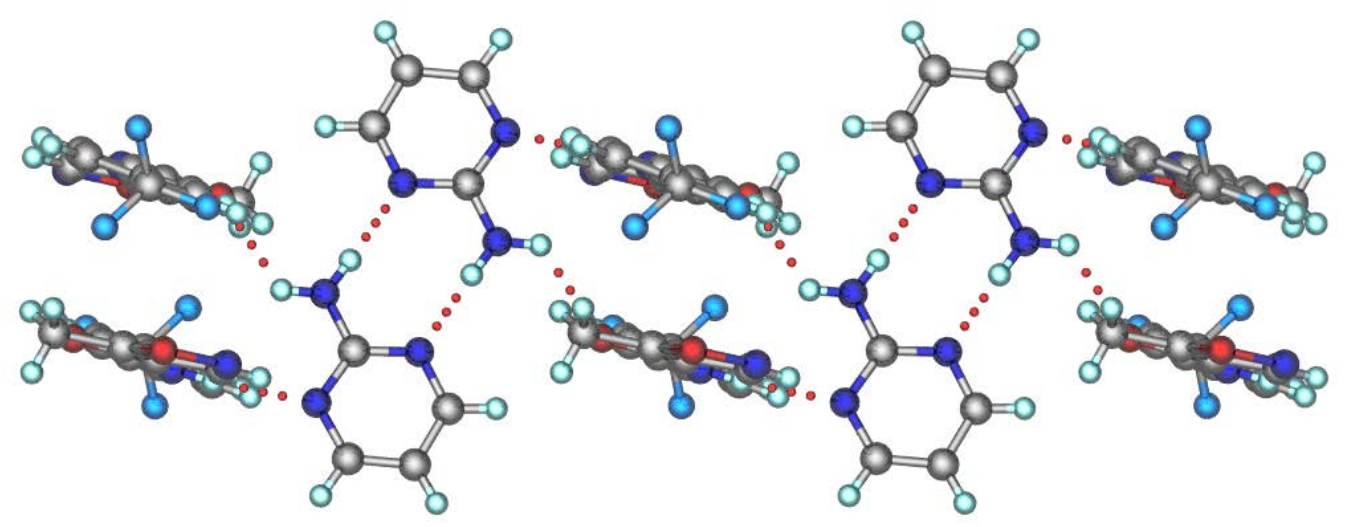

Figure 7. Crystal structure of the LEF-2APYM cocrystal viewed down the $(a+c) / 2$-axis showing the formation of 2APYM dimers and the continuous hydrogen-bonded sequence.

Comparison of the hydrogen bonding in the cocrystals of LEF suggests that, with the exception of the two cocrystals with 2PIC, the amide $\mathrm{C}=\mathrm{O}$ and $\mathrm{N}-\mathrm{H}$ of LEF is involved in formation of strong hydrogen bonds in all the cocrystals. While they take the center stage in the formation of extended hydrogen bonded chains of LEF molecules in the LEF-3HBA cocrystal, they link the strands of hydrogen bonded PG molecules in LEF-PG cocrystal. The dimers of 2APYM molecules are interconnected through LEF in the LEF-2APYM cocrystal. Interestingly, in the case of the cocrystals with 2PIC (stoichiometric variants), the ionic coformer (2PIC) selfassembles on its own via charge-assisted $\mathrm{N}^{+}-\mathrm{H} \cdots \mathrm{O}^{-}$hydrogen bonds and forms a dimeric motif in LEF-2PIC cocrystal and extended hydrogen bonded strands in LEF-2PIC (1:2) cocrystal. The second carboxylate $\mathrm{O}^{-}$forms an $\mathrm{N}-\mathrm{H} \cdots \mathrm{O}^{-}$hydrogen bond with the amide $\mathrm{N}-\mathrm{H}$ of LEF. In both cases, the absence of additional potential hydrogen bonding donor necessitates the amide $\mathrm{C}=\mathrm{O}$ to form auxiliary $\mathrm{C}-\mathrm{H} \cdots \mathrm{O}$ interactions with the adjacent 2PIC molecule. The ability of the ionic 2PIC molecule to form different hydrogen bonding modes and the fact that they are stabilised by the auxillary $\mathrm{C}-\mathrm{H} \cdots \mathrm{O}$ interactions facilitates the formation of these stoichiometric variants. Attempts to identify alternative stoichiometry for the other cocrystals using both grinding and solution-based techniques were not yet successful. 
Thermal Analysis. Thermal properties of LEF, cocrystals, and physical mixtures were analysed using DSC (Figure 8). TGA analysis was conducted to detect any trace amounts of solvent or water present in the bulk samples of cocrystals. It has been reported that both polymorphs of LEF show an identical melting behavior at $165^{\circ} \mathrm{C}$, with Form I showing an extra endotherm at $127^{\circ} \mathrm{C}$, which was ascribed to a phase transformation from Form I to Form II. ${ }^{24}$ The bulk LEF sample used in the present study is a mixture of Forms I and II. As shown in Figure 8, the thermal behavior of the bulk LEF is similar to Form I and shows an endothermic phase transition before melting. However, the phase transition occurs at a lower temperature $\left(110^{\circ} \mathrm{C}\right)$ than it was observed in the case of pure Form I $\left(127^{\circ} \mathrm{C}\right)$. DSC thermogram of all the cocrystals, except the LEF-2APYM, showed melting point lower than the melting point of LEF $\left(165.6^{\circ} \mathrm{C}\right.$, Table 1$)$, with modification of the melting point of an API a characteristic trait observed in cocrystals and commonly attributed to the effect of coformer melting point. ${ }^{38}$ In the case of LEF-2APYM cocrystal, the melting point lies between the melting point of LEF and 2APYM. A recent survery of melting point data of the reported cocrystals suggested that about $50 \%$ of the cocrystals showed melting point in between the melting point of the cocrystal constituents, while a lower cocrystal melting point is seen in fewer than $40 \% \cdot \frac{39}{}$ DSC thermograms of the corresponding physical mixtures of LEF cocrystals showed two endotherms closer to the melting point of lower melting constituent, indicating an impact of the other consituent as an impurity on the melting point (see Supporting Information, Figures S17-21). In the case of the physical mixture of LEF and 3HBA, the thermogram shows an endotherm at 110 ${ }^{\circ} \mathrm{C}$, indicative of the phase transformation of LEF Form I to II, followed by a single melting endotherm at $148{ }^{\circ} \mathrm{C}$ (Figure S18). Interestingly, the observed melting point of the physical mixture is lower than the melting point of the LEF-3HBA cocrystal $\left(153^{\circ} \mathrm{C}\right.$, Table 1$)$. We presume that the phyical mixture of LEF and 3HBA forms a eutectic composition upon heating. A more 
detailed analysis of this thermal behaviour is underway. TGA data of all the cocrystals showed no mass loss before their corresponding melting point seen in DSC, which indicates that the cocrystals are free from solvent/water inclusion in their crystal lattices (see Supporting Information, Figures S22-27).

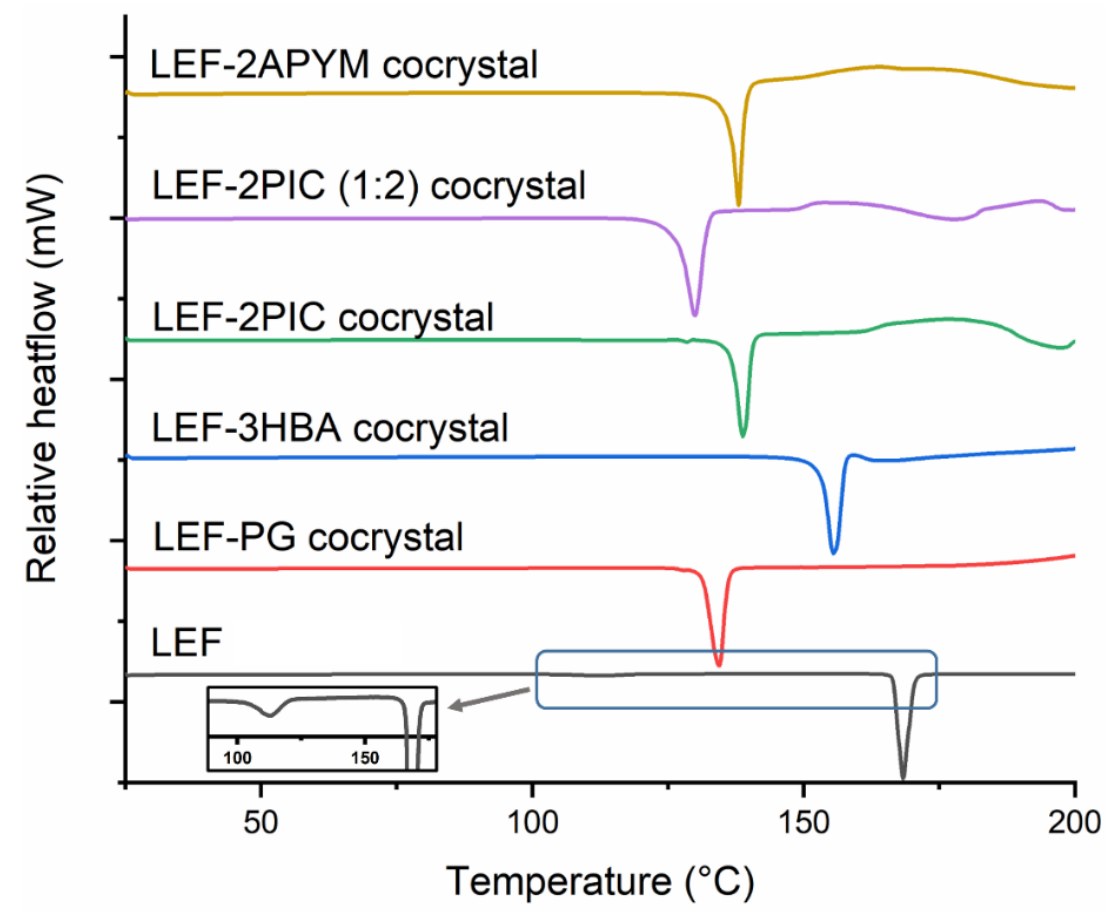

Figure 8. DSC thermograms of LEF and cocrystals.

Table 1. Melting point of LEF cocrystals and coformers

\begin{tabular}{llll}
\hline Cocrystal & Melting point $\left({ }^{\circ} \mathrm{C}\right)$ & Coformer & Melting point $\left({ }^{\circ} \mathrm{C}\right)$ \\
\hline LEF & 165.6 & - & - \\
LEF-PG & 130.7 & PG & 132.5 \\
LEF-3HBA & 153.1 & 3 HBA & 201.4 \\
LEF-2PIC & 135.4 & 2PIC & 137.4 \\
LEF-2PIC (1:2) & 125.4 & - & - \\
LEF-2APYM & 135.8 & 2APYM & 124.8
\end{tabular}


Stability Analysis. Stability of the cocrystals LEF-PG, LEF-3HBA, LEF-2PIC and LEF-2PIC 1:2 was evaluated, with respect to the parent API, under accelerated storage condition $\left(40{ }^{\circ} \mathrm{C}, 75 \%\right.$ RH) over a period of 13 weeks. PXRD data showed that all samples remained stable over the course of the testing period. Peaks associated with LEF were not observed in the PXRD patterns of the cocrystals throughout the testing period, indicating that there was no sign of cocrystal dissociation. The only minor stability issue was a slight loss of crystallinity in the LEF-2PIC cocrystal after 13 weeks. There was broadening in characteristic peaks at $2 \theta$ values of $8.9,14.8$ and 15.0 but the peak positions remained consistent (see Supporting Information, Figures S28-33).

The stability of LEF and cocrystals was also measured under slurry conditions. Excess powder samples were suspended in ultrapure water and stirred for 24 hours at $37^{\circ} \mathrm{C}$, then filtered and dried for PXRD analysis. The results for the LEF-PG, LEF-2PIC and LEF-2PIC 1:2 cocrystals showed crystalline peaks corresponding to LEF Form I in their respective post-slurry PXRD patterns (Figure 9), which suggest that these cocrystals dissociate in slurry and the more soluble PG and 2PIC coformers dissolve in solution and leave the poorly soluble LEF remaining as solid. On the other hand, the LEF-3HBA cocrystal remained stable in slurry, as is confirmed by the near perfect match of PXRD patterns before and after the slurry test. Slurrying bulk LEF (a mixture of Forms I and II) showed conversion of Form II to I, which was confirmed by the close similarity of the PXRD patterns of the post slurry sample and Form I of LEF (Figure 9).

Overall stability results show that cocrystallisation of LEF with the coformers did not impact upon stability under accelerated storage. While the cocrystals with PG and 2PIC showed dissociation under slurry condition, LEF-3HBA cocrystal remained stable. As mentioned previously, CSD interaction searching did not demonstrate the propensity of LEF to form carboxylic acid $\cdots \mathrm{N}(\mathrm{ar})$ heterosynthon, however $\mathrm{COOH}$ functional group was considered due to 
the reported abundance, strength and preference for synthon formation over other possible motifs such as acid-acid dimers. ${ }^{40}$ This could explain the greater stability of the LEF-3HBA under slurry condition, as the formation of stronger acid $\cdots \mathrm{N}$ (isoxazole) hydrogen bonds serves to maintain the crystal lattice, with such hydrogen bonds absent in the other cocrystals.
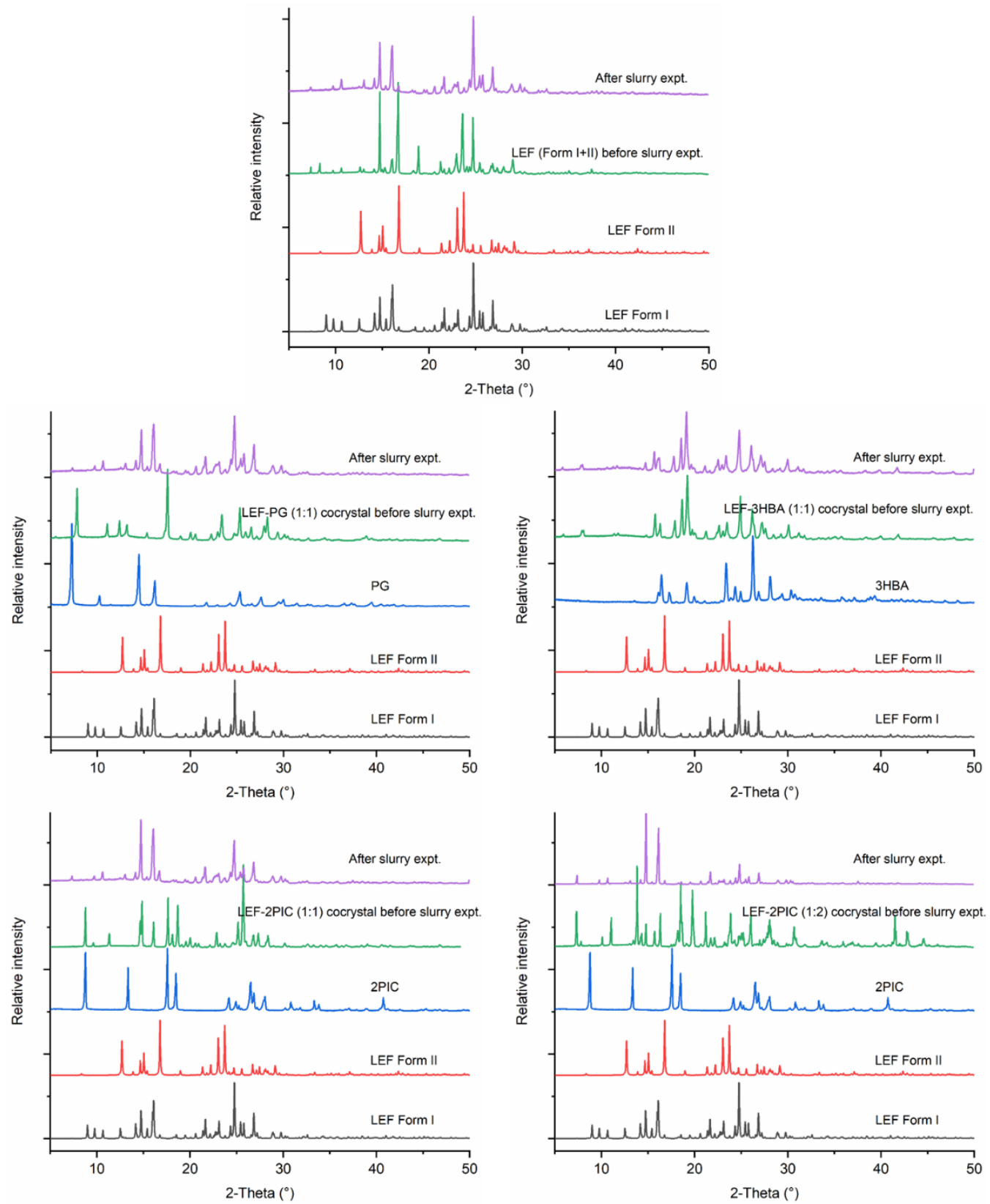

Figure 9. PXRD patterns of LEF cocrystals obtained under slurry condition, with reference to cocrystal before slurry experiments, LEF and the coformers. 
Solubility and Dissolution Rate. The impact of cocrystallisation on solubility and dissolution rate of LEF was evaluated. Solubility of the samples LEF and the stable cocrystal, LEF-3HBA, were measured using the shake-flask method. ${ }^{41}$ Prior stability results showed that the cocrystals LEFPG, LEF-2PIC and LEF-2PIC (1:2) were unstable under slurry condition therefore their true solubility is not reflected in these calculations. Apparent solubility of these samples was calculated by extracting the gradient of the linear portion of their respective dissolution rate plots. ${ }^{42}$ Table 2 shows the solubility values of the cocrystals, and their improvement with respect to that of the solubility of LEF (Form I). The greatest improvement in solubility is seen in the LEF-PG and LEF2PIC cocrystals, both of which have been shown to dissociate in solution. This improvement is partially expected as dissociable cocrystals are reported to possess higher solubility than their stable counterparts. $\frac{43}{}$ The stable and non-dissociating cocrystal (LEF-3HBA) also shows a slight improvement in solubility compared to LEF. The improved solubility of the LEF cocrystals could be ascribed to the higher solubility of the coformers. All the coformers possess higher aqueous solubility (PG, 3HBA and 2PIC: 625, 7.57, and $50 \mathrm{gL}^{-1}$, respectively) ${ }^{44}$ than LEF, with cocrystal solubility reported to be directly proportional to its constituents. $\frac{45}{4}$

Table 2. Solubility of LEF and cocrystals and the improvement in cocrystal solubility compared to LEF, calculated as a ratio of cocrystal solubility:LEF solubility.

\begin{tabular}{lll}
\hline Sample & Solubility/mgL & Solubility Improvement \\
\hline LEF (Form I) & $31.65 \pm 0.38$ & 1 \\
LEF-PG & $44.63 \pm 1.90^{\mathrm{a}}$ & 1.41 \\
LEF-3HBA & $36.58 \pm 3.44$ & 1.16 \\
LEF-2PIC & $40.11 \pm 1.04^{\mathrm{a}}$ & 1.27 \\
LEF-2PIC 1:2 & $34.45 \pm 0.68^{\mathrm{a}}$ & 1.09 \\
${ }^{\mathrm{a} A p p a r e n t ~ s o l u b i l i t y ~}$ & & \\
\hline
\end{tabular}


Dissolution rate measurements were performed by powder and intrinsic dissolution experiments in ultrapure water. An analysis of the IDR results indicated that there was only a minimal dissolution of the samples, which resulted in no apparent difference in the dissolution rate of the cocrystals and LEF (see Supporting Information, Figure S34). Therefore, special emphasis is given to the evaluation of powder dissolution rate of LEF, cocrystals and corresponding physical mixtures.

As shown in Figure 10, LEF cocrystals showed improved powder dissolution than the parent LEF. High supersaturation levels of cocrystals can be maintained even after $5 \mathrm{~h}$ with the LEF-2PIC cocrystal showing the highest supersaturation level. The observed supersaturation level of cocrystals and LEF at $5 \mathrm{~h}$ is in the order of LEF-2PIC $>$ LEF-PG $=$ LEF-3HBA $>$ LEF-2PIC (1:2) > LEF. Interestingly, initial dissolution rate of the physical mixtures is strikingly similar to the cocrystals, however as the dissolution progresses, the dissolution of all the physical mixtures remains similar to the parent LEF and reaches plateau at almost the same time. The physical mixture of LEF-2PIC (1:2) shows lower dissolution than the parent LEF, which is also in line with the cocrystal dissolution with the corresponding cocrystal (LEF-2PIC (1:2)) showing the lowest dissolution among the cocrystals of LEF.

All the cocrystals showed an improved dissolution rate in accordance with their increased solubility, however, the dissolution rate do not follow the same trend as that of their solubility. The LEF-2PIC cocrystal showed the fastest dissolution rate amongst all samples, whereas LEFPG is the most soluble cocrystal. It must be noted that the solubility values of the dissociable cocrystals were calculated from the initial linear portion of the dissolution curves (apparent solubility) at which kinetics dominate the dissolution rate and are thus not wholly representative of the thermodynamically-dependent equilibrium solubility. This is apparent as although LEF- 
3HBA shows a lesser improvement in calculated solubility than LEF-PG and LEF-2PIC cocrystals, its dissolution rate is nonetheless similarly enhanced compared to the parent API, suggesting the kinetics of dissolution are more distinct in these cocrystals.
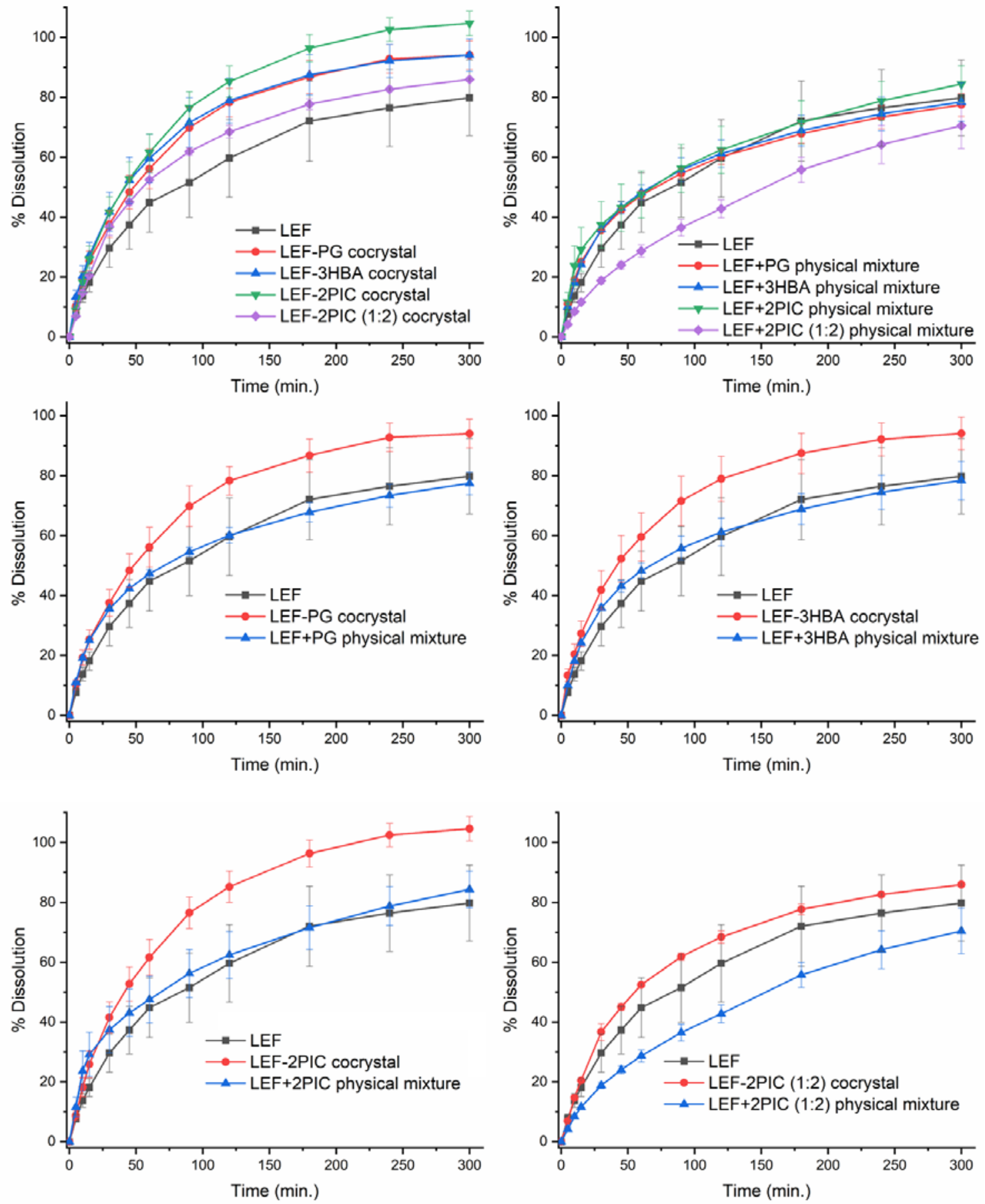

Figure 10. Dissolution rate profile for LEF and cocrystals in ultrapure water. 


\section{CONCLUSIONS}

Leflunomide, a BCS Class II antirheumatic drug, has its bioavailability limited by poor aqueous solubility and dissolution rate. Through detailed analysis of its structure and intermolecular interaction capabilities, using Isostar and Mercury, a range of favourable functional groups for its cocrystallisation, and a selection of coformers possessing these, have been identified. This rational selection of coformers has resulted in five new cocrystals of LEF. Crystal structure of the cocrystals has been determined and described in detail, with their intermolecular interactions highlighted with respect to the CSD interaction analysis. Their observed interactions display a number of supramolecular synthons previously recognised through prediction methods as promising interactions for LEF, such as amine $\cdots$ amide, acid $\cdots$ amide and acid $\cdots$ isoxazole.

Thermal analysis revealed that all the cocrystals melt at a temperature lower than the melting point of their respective components, except that melting point of the LEF-2APYM cocrystal lies between the melting point of its constituents. Physicochemical properties, such as stability, solubility and dissolution rate have been evaluated for the four pharmaceutically acceptable cocrystals_-LEF-PG, LEF-3HBA, LEF-2PIC and LEF-2PIC (1:2). LEF-3HBA cocrystal was the only cocrystal found to be stable under both accelerated and slurry conditions, as LEF-PG and both LEF-2PIC cocrystals showed signs of dissociation when slurried for 24 hours. This dissociation most likely allowed for the LEF-PG and LEF-2PIC cocrystals to greater improvement in apparent aqueous solubility of all samples, while LEF-3HBA cocrystal shows only a marginal improvement. Both LEF and cocrystals showed poor dissolution in IDR experiments, which resulted in all samples showing a similar dissolution patterns and hampered differentiation of their IDRs. Powder dissolution profiles of LEF, cocrystals and physical mixtures were analysed in detail. Notably, the physical mixtures showed either similar or lower dissolution 
rate than the parent LEF. On the other hand, the dissolution rate of the cocrystals was found to be higher than the parent LEF. Collation of all results for the four pharmaceutically acceptable cocrystals indicates that the coformers chosen in this study have showed an improvement in solubility and dissolution rate, albeit marginal. Given the availability of such a wide range of coformers and the ability of LEF to form cocrystals, cocrystallisation can be considered a viable route for development of novel LEF formulations.

\section{ASSOCIATED CONTENT}

Supporting Information. Detailed description and results of interaction searches used, list of potential coformers generated and those used in screening process, PXRD patters (from screening, slurry and stability tests), TGA analysis, DSC tehrmograms of physical mixtures, crystal structure parameters, hydrogen bond tables, and TGA thermograms). This material is available free of charge via the Internet at http://pubs.acs.org.

\section{AUTHOR INFORMATION}

\section{Corresponding Author}

*(S.A.) E-mail: srinivasulu_aitipamula@ices.a-star.edu.sg

\section{Notes}

The authors declare no competing financial interest.

\section{Author Contributions}


The manuscript was written through contributions of all authors. All authors have given approval to the final version of the manuscript.

\section{ACKNOWLEDGMENTS}

This research was funded by the Science and Engineering Research Council of A*STAR (Agency

for Science, Technology and Research), Singapore. Joseph Cadden thanks A*STAR for a research

fellowship jointly funded by the A*STAR Research Attachment Programme (ARAP) and The

University of Southampton.

\section{REFERENCES}

1. $\quad$ Al Qaraghuli, M. M.; Alzahrani, A. R.; Niwasabutra, K.; Obeid, M. A.; Ferro, V. A., Where traditional drug discovery meets modern technology in the quest for new drugs. Annals of Pharmacology and Pharmaceutics 2017, 2, 1-5.

2. $\quad$ Amidon, G. L.; Lennernas, H.; Shah, V. P.; Crison, J. R., A theoretical basis for a biopharmaceutic drug classification: the correlation of in vitro drug product dissolution and in vivo bioavailability. Pharm. Res. 1995, 12, 413-20.

3. Kasim, N. A.; Whitehouse, M.; Ramachandran, C.; Bermejo, M.; Lennernäs, H.; Hussain, A. S.; Junginger, H. E.; Stavchansky, S. A.; Midha, K. K.; Shah, V. P.; Amidon, G. L., Molecular properties of WHO essential drugs and provisional biopharmaceutical classification. Mol. Pharm. 2004, 1, 85-96.

4. Thayer, A. M., FINDING SOLUTIONS. Chem. Eng. News 2010, 88, 13-18.

5. $\quad$ Sai Gouthami, K.; Kumar, D.; Thipparaboina, R.; Chavan, R. B.; Shastri, N. R., Can crystal engineering be as beneficial as micronisation and overcome its pitfalls?: A case study with cilostazol. Int. J. Pharm. 2015, 491, 26-34.

6. $\quad$ Cheow, W. S.; Kiew, T. Y.; Yang, Y.; Hadinoto, K., Amorphization Strategy Affects the Stability and Supersaturation Profile of Amorphous Drug Nanoparticles. Mol. Pharm. 2014, 11, 1611-1620.

7. Williams, H. D.; Trevaskis, N. L.; Charman, S. A.; Shanker, R. M.; Charman, W. N.; Pouton, C. W.; Porter, C. J., Strategies to address low drug solubility in discovery and development. Pharmacol. Rev. 2013, 65, 315-499.

8. Sareen, S.; Mathew, G.; Joseph, L., Improvement in solubility of poor water-soluble drugs by solid dispersion. Int. J. Pharm. Investig. 2012, 2, 12-17.

9. $\quad$ Aitipamula, S.; Banerjee, R.; Bansal, A. K.; Biradha, K.; Cheney, M. L.; Choudhury, A. R.; Desiraju, G. R.; Dikundwar, A. G.; Dubey, R.; Duggirala, N.; Ghogale, P. P.; Ghosh, S.; Goswami, P. K.; Goud, N. R.; Jetti, R. R. K. R.; Karpinski, P.; Kaushik, P.; Kumar, D.; Kumar, V.; Moulton, B.; Mukherjee, A.; Mukherjee, G.; Myerson, A. S.; Puri, V.; Ramanan, A.;

Rajamannar, T.; Reddy, C. M.; Rodriguez-Hornedo, N.; Rogers, R. D.; Row, T. N. G.; Sanphui, P.; Shan, N.; Shete, G.; Singh, A.; Sun, C. C.; Swift, J. A.; Thaimattam, R.; Thakur, T. S.; Kumar Thaper, R.; Thomas, S. P.; Tothadi, S.; Vangala, V. R.; Variankaval, N.; Vishweshwar, P.; 
Weyna, D. R.; Zaworotko, M. J., Polymorphs, Salts, and Cocrystals: What's in a Name? Cryst. Growth Des. 2012, 12, 2147-2152.

10. Almarsson, O.; Zaworotko, M. J., Crystal engineering of the composition of pharmaceutical phases. Do pharmaceutical co-crystals represent a new path to improved medicines? Chem. Commun. 2004, 1889-96.

11. Bolla, G.; Nangia, A., Pharmaceutical cocrystals: walking the talk. Chemical Communications 2016, 52, 8342-60.

12. Aitipamula, S.; Chow, P. S.; Tan, R. B. H., Trimorphs of a pharmaceutical cocrystal involving two active pharmaceutical ingredients: potential relevance to combination drugs. CrystEngComm 2009, 11, 1823-1827.

13. Duggirala, N. K.; Perry, M. L.; Almarsson, Ö.; Zaworotko, M. J., Pharmaceutical cocrystals: along the path to improved medicines. Chem. Commun. 2016, 52, 640-655.

14. National Geographic Society, Bird evolution. In.

15. Morissette, S. L.; Almarsson, O.; Peterson, M. L.; Remenar, J. F.; Read, M. J.; Lemmo, A. V.; Ellis, S.; Cima, M. J.; Gardner, C. R., High-throughput crystallization: polymorphs, salts, co-crystals and solvates of pharmaceutical solids. Adv. Drug Delivery Rev. 2004, 56, 275-300.

16. Galek, P. T. A.; Pidcock, E.; Wood, P. A.; Bruno, I. J.; Groom, C. R., One in half a million: a solid form informatics study of a pharmaceutical crystal structure. CrystEngComm 2012, 14, 2391-2403.

17. Groom, C. R.; Bruno, I. J.; Lightfoot, M. P.; Ward, S. C., The Cambridge Structural Database. Acta Crystallographica Section B 2016, 72, 171-179.

18. Delori, A.; Galek, P. T. A.; Pidcock, E.; Patni, M.; Jones, W., Knowledge-based hydrogen bond prediction and the synthesis of salts and cocrystals of the anti-malarial drug pyrimethamine with various drug and GRAS molecules. CrystEngComm 2013, 15, 2916-2928. 19. Zhou, L.; Dodd, S.; Capacci-Daniel, C.; Garad, S.; Panicucci, R.; Sethuraman, V., Cocrystal formation based on structural matching. Eur. J. Pharm. Sci. 2016, 88, 191-201.

20. Mapp, L. K.; Coles, S. J.; Aitipamula, S., Design of Cocrystals for Molecules with Limited Hydrogen Bonding Functionalities: Propyphenazone as a Model System. Cryst. Growth Des. 2017, 17, 163-174.

21. Mapp, L. K.; Coles, S. J.; Aitipamula, S., Novel solid forms of lonidamine: crystal structures and physicochemical properties. CrystEngComm 2017, 19, 2925-2935.

22. Rozman, B., Clinical pharmacokinetics of leflunomide. Clin. Pharmacokinet. 2002, 41, 421-430.

23. El-Sayyad, N. M. E.-M.; Badawi, A.; Abdullah, M. E.; Abdelmalak, N. S., Dissolution enhancement of leflunomide incorporating self emulsifying drug delivery systems and liquisolid concepts. Bulletin of Faculty of Pharmacy, Cairo University 2017, 55, 53-62.

24. Vega, D.; Petragalli, A.; Fernández, D.; Ellena, J. A., Polymorphism on leflunomide: Stability and crystal structures. J. Pharm. Sci. 2006, 95, 1075-1083.

25. Desiraju, G. R., Supramolecular synthons in crystal engineering - a new organic synthesis. Angew. Chem., Int. Ed. Engl. 1995, 34, 2311-27.

26. Somphon, W. H., K. J., Crystal growth and physical characterization of picolinic acid cocrystallized with dicarboxylic acids. J. Cryst. Growth 2013, 362, 252-258.

27. Mashhadi, S. M. A. Y., U.; Bhatti, M. H.; Tahir, M. N, Isoniazid Cocrystals with AntiOxidant Hydroxy Benzoic Acids. J. Mol. Struct. 2014, 1076, 446.452.

28. Rigaku Oxford Diffraction, C. S. s., version 1.171.38.46, Rigaku Corporation, Oxford, UK, 2018. CrysAlisPro Software system, version 1.171.38.46; 2018. 
29. Sheldrick, G. M., Acta Crystallographica Section A 2008, 64, 112-122.

30. Sheldrick, G. M., Acta Crystallographica Section C 2015, 71, 3-8.

31. Dolomanov, O. V., Bourhis, L. J., Gildea, R. J., Howard, J. A. K. \& Puschmann, H., J. Appl. Cryst. 2009, 42, 339-341.

32. Spek, A. L., Acta Crystallographica Section D 2009, 65, 148-155.

33. Barbour, L. J., X-Seed - A Software Tool for Supramolecular Crystallography. Journal of Supramolecular Chemistry 2001, 1, 189-191.

34. Bruno, I. J.; Cole, J. C.; Lommerse, J. P. M.; Rowland, R. S.; Taylor, R.; Verdonk, M. L., IsoStar: A library of information about nonbonded interactions. J. Comput. Aided Mol. Des. 1997, 11, 525-537.

35. Macrae, C. F.; Bruno, I. J.; Chisholm, J. A.; Edgington, P. R.; McCabe, P.; Pidcock, E.; Rodriguez-Monge, L.; Taylor, R.; van de Streek, J.; Wood, P. A., Mercury CSD 2.0 - new features for the visualization and investigation of crystal structures. J. Appl. Crystallogr. 2008, 41, 466-470.

36. Shattock, T. R.; Arora, K. K.; Vishweshwar, P.; Zaworotko, M. J., Hierarchy of Supramolecular Synthons: Persistent Carboxylic Acid $\cdots$ Pyridine Hydrogen Bonds in Cocrystals That also Contain a Hydroxyl Moiety. Cryst. Growth Des. 2008, 8, 4533-4545.

37. Weyna, D. R.; Shattock, T.; Vishweshwar, P.; Zaworotko, M. J., Synthesis and Structural Characterization of Cocrystals and Pharmaceutical Cocrystals: Mechanochemistry vs. Slow Evaporation from Solution. Crystal Growth \& Design 2009, 9, 1106-1123.

38. Stanton, M. K.; Bak, A., Physicochemical Properties of Pharmaceutical Co-Crystals: A Case Study of Ten AMG 517 Co-Crystals. Crystal Growth \& Design 2008, 8, 3856-3862.

39. Schultheiss, N.; Newman, A., Pharmaceutical Cocrystals and Their Physicochemical Properties. Cryst. Growth Des. 2009, 9, 2950-2967.

40. Kavuru, P.; Aboarayes, D.; Arora, K. K.; Clarke, H. D.; Kennedy, A.; Marshall, L.; Ong, T. T.; Perman, J.; Pujari, T.; Wojtas, L.; Zaworotko, M. J., Hierarchy of Supramolecular Synthons: Persistent Hydrogen Bonds Between Carboxylates and Weakly Acidic Hydroxyl Moieties in Cocrystals of Zwitterions. Cryst. Growth Des. 2010, 10, 3568-3584.

41. Glomme, A.; Marz, J.; Dressman, J. B., Comparison of a miniaturized shake-flask solubility method with automated potentiometric acid/base titrations and calculated solubilities. J. Pharm. Sci. 2005, 94, 1-16.

42. $\quad$ Fagerberg, J. H.; Tsinman, O.; Sun, N.; Tsinman, K.; Avdeef, A.; Bergström, C. A. S., Dissolution Rate and Apparent Solubility of Poorly Soluble Drugs in Biorelevant Dissolution Media. Mol. Pharm. 2010, 7, 1419-1430.

43. Reddy, L. S.; Bethune, S. J.; Kampf, J. W.; RodriÌ Hgoredo, N. r., Cocrystals and Salts of Gabapentin: pH Dependent Cocrystal Stability and Solubility. Crystal Growth \& Design 2008, 9, 378-385.

44. Nordström, F. L.; Rasmuson, Å. C., Polymorphism and thermodynamics of mhydroxybenzoic acid. Eur. J. Pharm. Sci. 2006, 28, 377-384.

45. Good, D. J.; Rodriguez-Hornedo, N., Solubility Advantage of Pharmaceutical Cocrystals. Crystal Growth \& Design 2009, 9, 2252-2264. 


\section{For Table of Contents Use Only}

\section{Cocrystals of Leflunomide: Design, Structural and Physicochemical Evaluation}

Joseph Cadden, Wim T. Klooster, Simon J. Coles and Srinivasulu Aitipamula

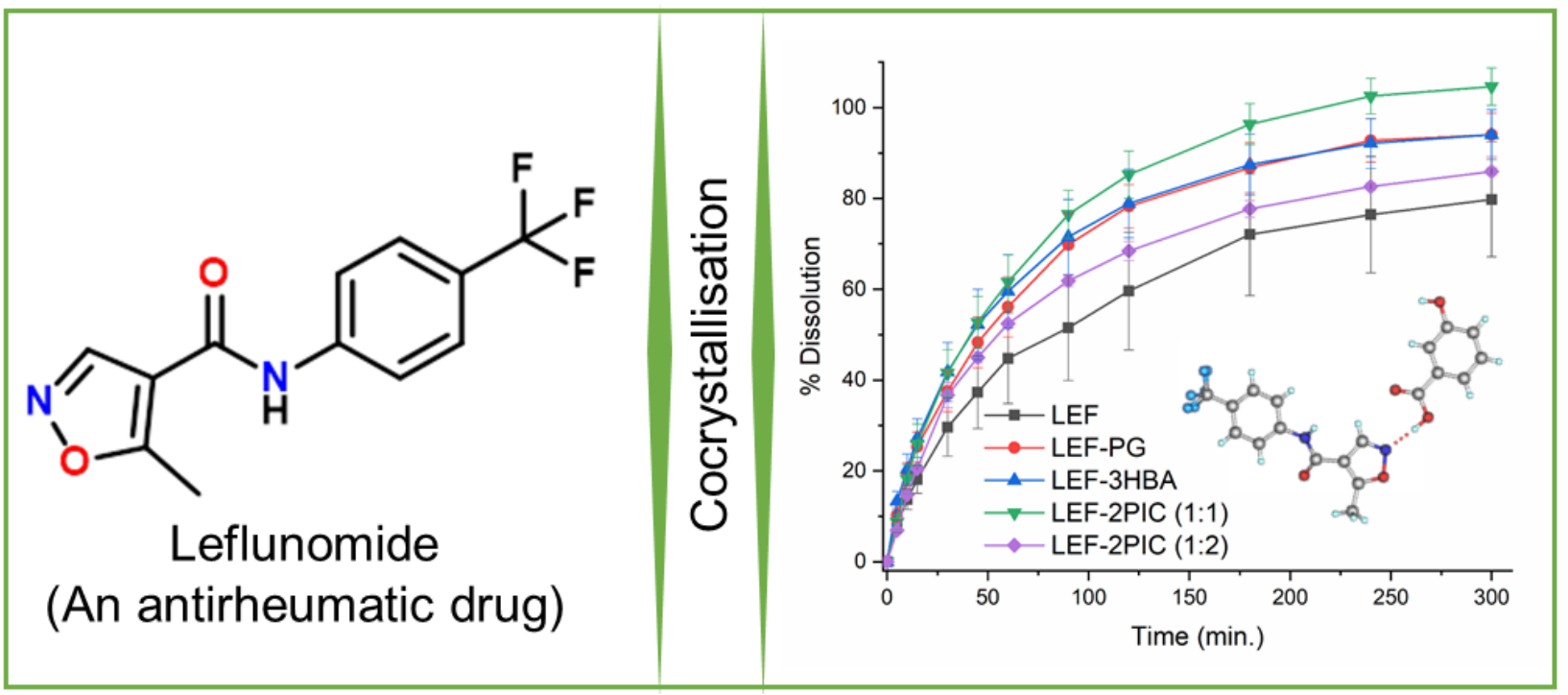

Leflunomide is an immunosuppressive disease-modifying antirheumatic drug whose bioavailability is limited by its poor solubility and dissolution rate. We report five cocrystals of leflunomide and their characterisation by various analytical techniques. Cocrystals showed a marginal improvement in solubility of leflunomide, but maintained high drug supersaturation levels in dissolution experiments. In contrast, dissolution profiles of the constituent physical mixtures strikingly similar to the parent leflunomide. 